DRAFT VERSION OCTOBER 30, 2018

Preprint typeset using LTEX style emulateapj v. 11/10/09

\title{
PARTICLE MESH SIMULATIONS OF THE LYMAN $\alpha$ FOREST AND THE SIGNATURE OF BARYON ACOUSTIC OSCILLATIONS IN THE INTERGALACTIC MEDIUM
}

\author{
Martin White ${ }^{1,2}$, Adrian Pope $^{3,4,7}$, Jordan CARlson $^{1}$, Katrin Heitmann $^{3}$, Salman Habib $^{4}$, Patricia Fasel ${ }^{6}$, \\ DAVID DANIEL ${ }^{5}$, AND ZARIJA LUKIC ${ }^{4}$ \\ ${ }^{1}$ Department of Physics, University of California Berkeley, CA 94720 \\ 2 Department of Astronomy, University of California Berkeley, CA 94720 \\ ${ }^{3}$ ISR-1, Los Alamos National Laboratory, Los Alamos, NM 87545 \\ ${ }^{4}$ T-2, Los Alamos National Laboratory, Los Alamos, NM 87545 \\ ${ }^{5}$ CCS-1, Los Alamos National Laboratory, Los Alamos, NM 87545 \\ ${ }^{6}$ CCS-3, Los Alamos National Laboratory, Los Alamos, NM 87545 \\ 7 CCS-6, Los Alamos National Laboratory, Los Alamos, NM 87545 and \\ LA-UR 09-07334 \\ (Dated: October 30, 2018) \\ Draft version October 30, 2018
}

\begin{abstract}
We present a set of ultra-large particle-mesh simulations of the Lyman- $\alpha$ forest targeted at understanding the imprint of baryon acoustic oscillations (BAO) in the inter-galactic medium. We use 9 dark matter only simulations which can, for the first time, simultaneously resolve the Jeans scale of the intergalactic gas while covering the large volumes required to adequately sample the acoustic feature. Mock absorption spectra are generated using the fluctuating Gunn-Peterson approximation which have approximately correct flux probability density functions (PDFs) and small-scale power spectra. On larger scales there is clear evidence in the redshift space correlation function for an acoustic feature, which matches a linear theory template with constant bias. These spectra, which we make publicly available, can be used to test pipelines, plan future experiments and model various physical effects. As an illustration we discuss the basic properties of the acoustic signal in the forest, the scaling of errors with noise and source number density, modified statistics to treat mean flux evolution and mis-estimation, and non-gravitational sources such as fluctuations in the photo-ionizing background and temperature fluctuations due to HeII reionization.
\end{abstract}

Subject headings: methods: $N$-body simulations — cosmology: large-scale structure of universe

\section{INTRODUCTION}

Oscillations of the baryon-photon plasma in the early universe, also known as Baryon Acoustic Oscillations (BAO), imprint a distinct signature on the clustering of matter (Peebles \& Yu 1970; Sunyaev \& Zeldovich 1970) which provides a "standard ruler" by which we can measure the expansion history of the Universe (see Eisenstein \& Hu 1998; Meiksin et al. 1999 for a detailed description of the physics in modern cosmologies and Eisenstein et al. 2007 for a comparison of Fourier and configuration space pictures). These oscillations have been traditionally measured in the Cosmic Microwave Background (CMB; see Jarosik et al. 2010, for the latest results) but with the advent of new, large-volume galaxy redshift surveys BAO have been detected in galaxy clustering at low $z$ as well (Eisenstein et al. 2005; Cole et al. 2005; Hütsi 2006; Blake et al. 2007; Padmanabhan et al. 2007a; Percival et al. 2007, 2009).

In principle, the $\mathrm{BAO}$ technique becomes even more powerful as one moves to higher redshift, where there is much more volume available to be surveyed and where the acoustic scale is more deeply in the linear regime. This latter fact helps in two ways. First, the power spectrum or correlation function can be computed quite accurately with only linear perturbation theory once one specifies the baryon-to-photon ratio and matter-radiation ratio. These are both measured accurately from CMB acoustic peaks (see White \& Cohn 2002 for a review) so we have a template with which to fit the data. Second, the acoustic oscillations are less damped by non-linear evolution providing more modes which contain measurable signal.

Tracing the enormous volumes required with galaxies requires a heavy investment in telescope time. However, in principle any tracer of the mass field will do, including the neutral hydrogen in the inter-galactic medium (White 2003) or galaxies (Chang et al. 2008). Tracing neutral hydrogen in galaxies via its redshifted $21 \mathrm{~cm}$ emission is a key goal for proposed future radio telescopes. However, even with current technology it is relatively straightforward to obtain a low resolution spectrum of distant quasars and study the Lyman- $\alpha$ forest of absorption lines which map the neutral hydrogen along the line-of-sight. At $z \simeq 2-3$ the gas making up the inter-galactic medium (IGM) is thought to be in photo-ionization equilibrium, which results in a tight densitytemperature relation for the absorbing material with the neutral hydrogen density proportional to a power of the baryon density (Hui \& Gnedin 1997; Meiksin 2007). Since pressure forces are sub-dominant, the neutral hydrogen density closely traces the total matter density on large scales. The structure in QSO absorption thus traces, in a calculable way, slight fluctuations in the matter density of the universe back along the line-of-sight to the QSO, with most of the Lyman- $\alpha$ forest arising from over-densities of a few times the mean density.

Motivated by the upcoming Baryon Oscillation Spectroscopic Survey (BOSS; Schlegel et al. 2009, a part of SDSSIII), which will deliver an unprecedented number of quasar spectra probing the Lyman- $\alpha$ forest at $z \sim 2-3$, and access to the world's fastest supercomputer, "Roadrunner", we have produced a set of ultra-large particle-mesh simulations of cosmological structure formation to further investigate BAO- 
Lyman- $\alpha$ science. In this paper we describe our simulations, which simultaneously resolve the Jeans scale and the acoustic scale, and the construction of mock quasar spectra with properties close to those observed at $z \sim 2-3$ (Sec. 2). We present several examples to illustrate the use of these spectra in understanding BAO-Lyman- $\alpha$ science, describing the 2-point correlation function of the Lyman- $\alpha$ forest flux in Sec. 3, and the impact of two non-gravitational signals (fluctuations in the ionizing background and HeII reionization) in Sec. 4 . We conclude in Sec.5] In the hope that these spectra can be more generally useful, we have made them publicly available?.

\section{SIMULATIONS}

\subsection{Particle-mesh simulations}

The study of the BAO signal in the Lyman- $\alpha$ forest is difficult both analytically and numerically. Much of the signal comes from inter-galactic gas at near mean density, imposing strong requirements on the mass resolution of any simulation. The gas contains structure down to the Jeans scale, $\mathcal{O}(100 \mathrm{kpc})$, and resolving this structure while simulating the extremely large volumes required by BAO demands impressive dynamic range. As part of the "Roadrunner Universe" project (Habib et al. 2009) we have run a set of ultra-large particle-mesh simulations, in order to resolve the Jeans scale in a large cosmological volume.

In particular we have run 9 particle-mesh simulations of a flat $\Lambda$ CDM cosmology, with $\Omega_{M}=0.25, \Omega_{\Lambda}=0.75, h=0.72$, $n=0.97$ and $\sigma_{8}=0.8$ (i.e. model 0 of Heitmann et al. 2008, 2009). All of the simulations were started at $z=211$ from an initially regular Cartesian grid of particles, displaced according to the Zel'dovich approximation. Each simulation evolved $4,000^{3}$ particles in a $750 h^{-1} \mathrm{Mpc}$ box (particle mass $\left.5 \times 10^{8} h^{-1} M_{\odot}\right)$ computing the forces on a $4,000^{3}$ grid. For a description of the particle-mesh code, and code tests, see Habib et al. (2009). The correlation function of the mass at $z \simeq 2.4$ is shown in Figure 1, compared to the theoretical prediction.

These simulations are broadly similar to those reported in Slosar et al. (2009). However those simulations, which were focused on the large-scale BAO signal, did not properly resolve the small-scale power. While this should not bias the shape of the flux correlation function on large scales, it does affect the distribution of pixels and the large-scale bias, which in turn affects the signal-to-noise of any measurement. Further massaging of such low resolution spectra is thus required before they can be used for pipeline tests, to investigate signal-to-noise scaling, or as input to MonteCarlo simulations. The larger dynamic range in mass and force resolution of the Roadrunner simulations allows us to maintain high resolution (comparable to previous authors, e.g. Meiksin \& White 2001; McDonald 2003; Mandelbaum et al. 2003; McDonald et al. 2005) while simulating a relatively large computational volume. Not even our simulations can resolve $100 h^{-1} \mathrm{kpc}$ over the full $1.5 \mathrm{~h}^{-1} \mathrm{Gpc}$ simulated in Slosar et al. (2009), so we must compromise on either volume or resolution. We have chosen to maintain high force and mass resolution by running smaller boxes than in Slosar et al. (2009) and build up total volume by performing several realizations.

While the total volume can be enlarged by running many individual simulations, if each has too small a box there can

\footnotetext{
${ }^{1}$ http://mwhite.berkeley.edu
}

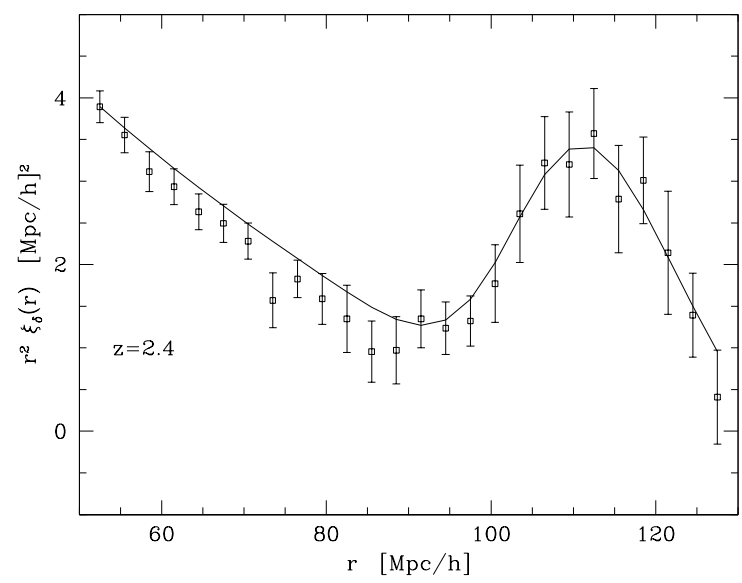

FIG. 1.- The real-space mass correlation function, $\xi_{\delta}(r)$, with bootstrap errors. The solid line shows the prediction of linear theory, convolved with a Gaussian of $3 h^{-1} \mathrm{Mpc}$ (see $\$ 3.2$ ).

be systematic effects introduced into the measurement of the acoustic scale. At the high redshifts of interest for Lyman$\alpha$ forest work the missing long-wavelength modes in a finite box simulation do not systematically alter the evolution of the existing modes — the fundamental mode of the box is well in the linear regime. However the finite support of the $k$-modes implied by periodicity may alter the correlation function on the scales of interest. To test for this we generated correlation functions by Fourier transforming power spectra sampled only on the $k$-grid allowed by periodic boxes of a range of sizes and compared them to the $L_{\text {box }} \rightarrow \infty$ limit. The largest effect is an almost scale independent suppression of $\xi(r)$ in the smaller boxes, but this becomes negligible once the box is larger than $500 h^{-1} \mathrm{Mpc}$. For our choice of $750 h^{-1} \mathrm{Mpc}$, the correlation function at $r \sim 10^{2} h^{-1} \mathrm{Mpc}$ is indistinguishable from that in a much larger box. Our choice of box size and resolution is thus adequate for studying the $\mathrm{BAO}$ signature in the Lyman- $\alpha$ forest in some detail.

Recently Norman et al. (2009) have reported fully hydrodynamic simulations covering slightly smaller volumes with slightly worse force and mass resolution than the simulations reported here. The advantage of these simulations is that they attempt to treat the baryonic physics more accurately, but with a concomitant increase in computational complexity and hence decrease in dynamic range.

\subsection{Mock spectra}

From the phase space data for the particles we produced 'skewers' through the simulation cube of density and line-ofsight velocity at $z=2.25,2.4,2.5$ and 2.75 . The skewers are distributed at random across the face of the cube, and run parallel to the sides of the box.

The density and velocity fields were smoothed with a filter of width $100 h^{-1} \mathrm{kpc}$ to approximate the effects of thermal pressure forces on the gas. Such a scheme clearly ignores much of the complexity of gas processes on small scales, but comparison with hydrodynamic simulations indicates it retains many of the features of interest for the Ly- $\alpha$ forest (see Meiksin 2007, for a review). For an isothermal gas at mean density the (comoving) Jeans filtering scale is

$$
k_{J}=\sqrt{\frac{3}{2}} \frac{H(z)}{(1+z) c_{s}}
$$


with $c_{s}=\left(5 / 3 k_{B} T / \widehat{m}\right)^{1 / 2}$ and $\widehat{m}=0.588 m_{H}$. However this is the appropriate smoothing scale only if the gas temperature scales as $(1+z)$. While the observational situation is uncertain, it appears that the gas temperature scales more slowly than this, and the comoving Jeans scale increases with time. For this reason Gnedin \& Hui (1998) argue for a smoothing scale set by the Jeans scale at an earlier epoch, specifically $\simeq 2$ times smaller than the Jeans scale at the time of observation. Modeling the filtering as a Gaussian and assuming $2 \times 10^{4} \mathrm{~K}$ gas (Ricotti et al. 2000; Schave et al. 2000; McDonald et al. 2001; Zaldarriaga et al. 2001a; Theuns et al. 2002; Lidz et al. 2009) gives $\sigma=0.14 h^{-1} \mathrm{Mpc}$ at $z=2$ and $\sigma=0.12 h^{-1} \mathrm{Mpc}$ at $z=3$. Given the uncertainties, and the approximate treatment of this physics, we shall assume $\sigma=100 h^{-1} \mathrm{kpc}$ in what follows.

For numerical reasons we used a spline kernel (Dehnen 2001) rather than a Gaussian. The spline kernel approximates a Gaussian well in the core, but vanishes identically beyond a range, $R$. A good match is found when $R=3.25 \sigma$, so we adopt $R=0.325 h^{-1} \mathrm{Mpc}\left(\sigma \simeq 100 h^{-1} \mathrm{kpc}\right)$ which is larger than our mesh scale and mean inter-particle spacing. We have checked that increasing the resolution does not appreciably change the spectra when the density field is smoothed on these scales, indicating that we are numerically converged.

For each of 22,500 randomly placed lines-of-sight per box the fluctuating Gunn-Peterson approximation (FGPA; Croft et al. 1998; Gnedin \& Hui 1998; Meiksin 2007) was used to generate skewers of optical depth with 4,000 pixels each. We assumed a temperature at mean density of $2 \times 10^{4}$ $\mathrm{K}$ (McDonald et al. 2001; Theuns et al. 2002) and equations of state running from $\gamma=0.5$ to $\gamma=1.5$. Different choices for the slope, even the inverted equation of state $(\gamma<1)$, quantitatively but not qualitatively change our conclusions. The optical depth included thermal broadening (assumed Gaussian) and skewers are generated both with and without peculiar velocities for the gas.

The optical depth was scaled so that the mean transmitted flux $\bar{F}=\langle\exp (-\tau)\rangle$ approximately matched that of the data compiled in Meiksin \& White (2004):

$$
-\ln \bar{F}=0.00211(1+z)^{3.7}
$$

valid for $1.2<z<4$. We impose this mean flux condition over the entire volume. As described later, we also generate skewers in which $\bar{F}$ changes across the volume to understand the effect of incomplete modeling of the mean flux evolution. Another way to set $\bar{F}$ is via the amplitude of the flux power spectrum. Conveniently, these two methods agreed quite well. For completeness, we also generate the skewers with darkmatter over-density only, so we can compare the flux statistics to those of the underlying mass.

We work throughout with relative fluctuations in the flux, $\delta_{F}=F(\hat{x}) / \bar{F}-1$, so our fundamental data set is $\delta_{F}(\vec{x})$ on 22,500 skewers of 4,000 pixels each per box. Our 9 simulations have $V \simeq 3.8\left(h^{-1} \mathrm{Gpc}\right)^{3}$ and cover approximately 1,000 sq.deg., or $10 \%$ of the coverage planned for BOSS. On the other hand the line-of-sight areal density $(\sim 200$ per sq.deg. at $z=2.5)$ is much larger than anticipated from BOSS. This allows us to study the impact of quasar number density on Lyman- $\alpha$ forest studies for future missions. Of course any observational program will likely analyze the data in small shells of redshiff ${ }^{2}$ over which the evolution of e.g. the mean

${ }^{2}$ Since our simulation boxes are dumped at constant time they can also

\begin{tabular}{ccccccc}
$z$ & $\lambda_{\alpha}$ & $\chi_{\alpha}$ & $\chi_{\beta}-\chi_{\alpha}$ & $H(z)$ & $d \lambda / d \chi$ & $d v / d \chi$ \\
\hline 1.75 & 3343.1 & 3506 & 590 & 244 & 0.99 & 89 \\
2.00 & 3647.0 & 3796 & 575 & 274 & 1.11 & 91 \\
2.25 & 3950.9 & 4055 & 561 & 305 & 1.24 & 94 \\
2.50 & 4254.8 & 4288 & 546 & 339 & 1.37 & 97 \\
2.75 & 4558.8 & 4499 & 531 & 373 & 1.51 & 100 \\
3.00 & 4862.7 & 4690 & 518 & 409 & 1.66 & 102
\end{tabular}

TABLE 1

SOME USEFUL CONVERSION FACTORS FOR OUR COSMOLOGY. FOR EACH REDSHIFT, $z$, WE LIST THE OBSERVED WAVELENGTH $\left[\lambda_{\alpha}\right]$ OF LY- $\alpha$ EMITTED AT THAT $z$, THE COMOVING DISTANCE TO THAT REDSHIFT

$\left[\chi_{\alpha}\right]$, THE EXTRA COMOVING DISTANCE TO LY- $\beta$ IF LY- $\alpha$ IS AT THAT $z$ $\left[\chi_{\beta}-\chi_{\alpha}\right]$, THE HUBBLE PARAMETER $[H(z)]$ AND CONVERSIONS BETWEEN DISTANCE AND WAVELENGTH $[d \lambda / d \chi]$ AND VELOCITY

$[d v / d \chi]$. Distances ARE MEASURED IN $h^{-1}$ MPC, VELOCITIES IN KM/S AND WAVELENGTHS IN $\AA$.

flux, is small. A survey such as BOSS should also be able to detect the evolution in $H(z)$ across the redshift range $2<z<3$ by the shift in the acoustic feature in velocity space.

\subsection{Properties of the simulated spectra}

At the fiducial redshift of our box, $z \sim 2.5$, the Ly- $\alpha$ feature is redshifted to approximately $4,000 \AA$, and the box has a velocity "length" of 73,000 km/s. Each of our mock spectra thus encompasses the full Ly- $\alpha$ to Ly- $\beta$ region for QSOs at $z \sim 2.5$ (Table 1). For $z \simeq 2-3$ a comoving $h^{-1} \mathrm{Mpc}$ is approximately equal to $1 \AA$ (Table 1 ), so with 4,000 pixels per spectrum, each simulation pixel is $0.2 h^{-1} \mathrm{Mpc}$ wide. For comparison each SDSS-III pixel is about $75 \mathrm{~km} / \mathrm{s}$ (or just under $1 h^{-1} \mathrm{Mpc}$ ), so our spectra are comparably well resolved.

We normalize the optical depths so that the mean transmitted flux matches observations. An additional constraint then comes from comparing the flux variance of the simulations and observations. For $\gamma=0.5-1.5$ we find $\sigma_{F}^{2}=0.1$, which is very comparable to the measurements reported in McDonald et al. (2000). Figure 2 shows the flux PDF in the simulations compared to that measured from high resolution spectra at $z \simeq 2.4$ in Kim et al. (2007). We see that the distribution of high and low absorption regions is approximately correct, indicating that the spectra could be useful for testing analysis pipelines and investigating the impact of systematic errors. While the discrepancies are larger than the quoted observational error bars, we note that our modeling could be improved and the flux PDF is notoriously difficult to measure observationally (especially in regions of low absorption). We have not attempted a more detailed comparison since the level of agreement will be sufficient for our purposes.

The line-of-sight power spectrum of the flux at $z \simeq$ 2.4, compared to the measurements of Croft et al. (2002), Kim et al. (2004) and McDonald et al. (2006), is shown in Figure 3. There is some tension between the flux PDF and the power spectrum in the preferred value of $\bar{F}$ : the flux PDF is better fit if we slightly raise $\bar{F}$, increasing the number of lines of sight with little or no absorption and decreasing the number with little or no transmission. The flux power spectrum is better fit if we lower $\bar{F}$, raising the amplitude of $P_{F}(k)$. For $\bar{F} \simeq 0.8$ the overall the level of agreement is good in both statistics, indicating that the relative amounts of power on different scales are approximately as observed in the

be thought of as effectively larger sky area of "thin" redshift slices. However the limited box size means different subregions of the boxes are not fully independent. 


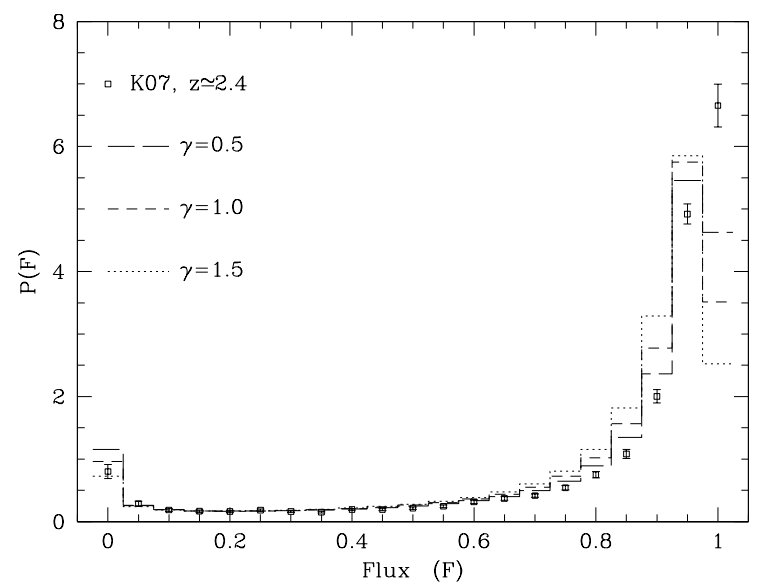

FIG. 2.- The flux PDF in simulations (lines) and as measured in high resolution spectra by Kim et al. (2007, squares). Overall the simulations do a fair job of describing the flux PDF, containing approximately the right distribution of low and high absorption regions.

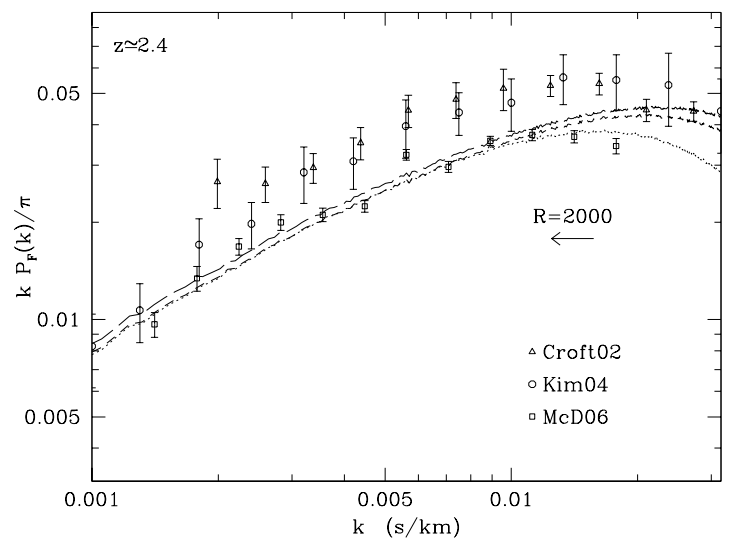

FIG. 3.- The (dimensionless) line-of-sight power spectrum of the flux measured from the simulations at $z \simeq 2.4$ and from Croft et al. (2002, triangles), Kim et al. (2004, circles) and McDonald et al. (2006, squares). As in Figure 2 line types indicate the assumed equation-of-state: $\gamma=0.5$ (longdashed), $\gamma=1.0$ (short-dashed) and $\gamma=1.5$ (dotted). The arrow indicates roughly the range of scales accessible from $R=2,000$ spectra such as will be produced by BOSS.

real Universe. We have chosen to match to the later, SDSSbased measurements of McDonald et al. (2006) rather than the slightly higher results of Croft et al. (2002); Kim et al. (2004). Our spectra thus contain approximately the right distribution of fluxes and approximately the right amount of small-scale structure, which acts as a source of "noise" in the measurement of the acoustic scale.

In what follows we will work with $\delta_{F}$, ignoring realworld issues such as continuum fitting or subtraction, damped Lyman- $\alpha$ systems and metal lines. We expect the damping wings and metal lines to be uncorrelated with the signal of interest, and so not produce a feature at the acoustic scale. Similarly, we will be looking at cross-correlation statistics between different lines-of-sight. Since several million QSO pairs contribute to the flux 2-point function, $\xi_{F}\left(r_{B A O}\right)$, the fluctuations will rapidly average to zero (Viel et al. 2002).

\section{TWO POINT STATISTICS}

\subsection{The correlation function}

We compute the density-density or flux-flux (cross-) correlation function as a direct sum over pixel pairs, omitting pixel pairs in the same line-of-sight. We will focus on isotropically averaged statistics - due to our limited volume we do not have a statistically significant detection of the quadrupole $\left[\xi_{2}\right]$ in the acoustic peak region 3 . We use $3 h^{-1} \mathrm{Mpc}$ bins, such that the effect of binning changes $\xi$ by less than $1 \%$ in the region of the acoustic peak. The binned correlation function for any model can be as easily computed as the unbinned correlation function, e.g.

$$
\xi_{F}\left(r_{i} ; \Delta r\right)=\int \frac{d^{3} k}{(2 \pi)^{3}} P_{F}(k) W_{i}(k)
$$

where $P_{F}$ is the flux power spectrum and

$$
W_{i}(k)=3 \frac{\sin \left(x_{+}\right)-\sin \left(x_{-}\right)-x_{+} \cos \left(x_{+}\right)+x_{-} \cos \left(x_{-}\right)}{x_{+}^{3}-x_{-}^{3}}
$$

with $x_{ \pm}=k(r \pm \Delta r / 2)$. As $\Delta r \rightarrow 0$ we have $W_{i} \rightarrow j_{0}(k r) \equiv$ $\sin (k r) /(k r)$. Larger $\Delta r$ leads to increased damping as $k \rightarrow \infty$ and a very slight increase in the degree of correlation between adjacent bins. For $\Delta r \rightarrow 0$, infinitesimal pixels and white noise, the error on a given $\xi_{F}(r)$ is infinite. The noise contribution to a binned statistic is however finite. If our $r$ bins are larger than our pixels (as in our case) then it is the bin width which tames the noise - any results on e.g. S/N are thus dependent on the bin size.

\subsection{Template}

In general the flux correlation function (Figure 4) can be related to the non-linear, redshift-space, mass correlation function, $\xi_{m}(r)$ as

$$
\xi_{F}(r)=B(r) \xi_{m}(r)+A(r)
$$

for some smooth functions $B(r)$ and $A(r)$.

A first approximation to $\xi_{m}$ is to take a multiple of the real-space mass correlation function predicted by linear theory. The dominant effect of non-linear clustering is to broaden the acoustic peak, with an amplitude that can be estimated from the rms Zel'dovich displacement (Bharadwai 1996; Eisenstein et al. 2007; Crocce \& Scoccimarro 2008; Matsubara 2008). At $z=2.5$ this is about $3 h^{-1} \mathrm{Mpc}$ in our cosmology, to be compared to the much larger intrinsic width of the acoustic feature (set by the diffusion, or Silk, damping scale: $12 h^{-1} \mathrm{Mpc}$ ). Figure 1 shows the mass correlation function measured in the simulations (with errors determined from bootstrap as described below) compared to this smoothed linear theory. The agreement is very good over a broad range of scales (recall the errors are highly correlated).

On the large scales of interest here redshift space distortions simply multiply the real-space, mass correlation function by $1+(2 / 3) f+(1 / 5) f^{2}-$ where $f \equiv d \ln \delta / d \ln a \simeq \Omega_{m}^{0.6} \simeq 1$ is the growth factor (Kaiser 1987; Hamilton 1992) - and apply smoothing of the same order as above. Adding these in quadrature we see non-linear evolution and redshift space distortions will only change the peak width by $\sim 5 \%$. A reasonable approximation to $\xi_{m}(r)$, therefore, is the linear theory, real-space, mass correlation function multiplied by a constant and convolved with a Gaussian of $4 h^{-1} \mathrm{Mpc}$.

\footnotetext{
${ }^{3}$ We do see evidence at smaller $r$ for a lower quadrupole-to-monopole ratio than in the case of the mass. This is expected due to the mapping from density to flux.
} 


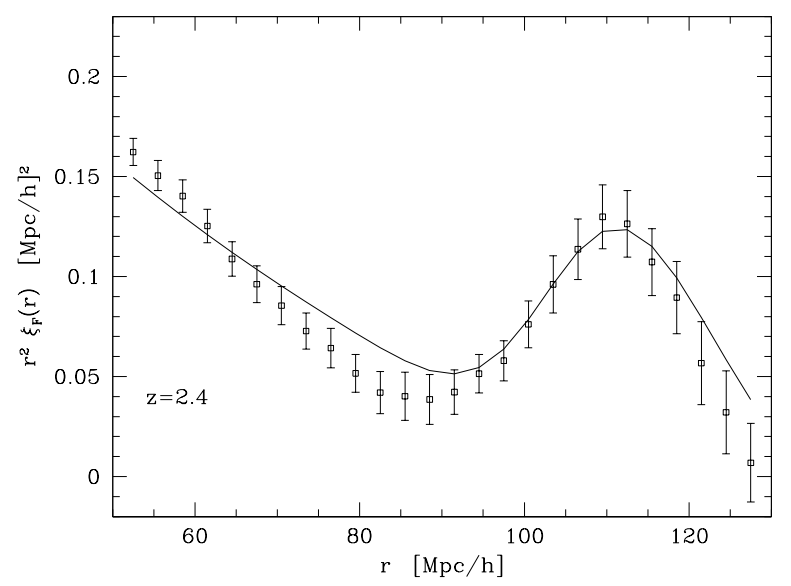

FIG. 4.- The redshift space flux correlation function, $\xi_{F}$, with bootstrap errors. The solid line shows linear theory, convolved with a Gaussian and multiplied by a constant bias of $b^{2}=0.2^{2}$ (see Figure 5.

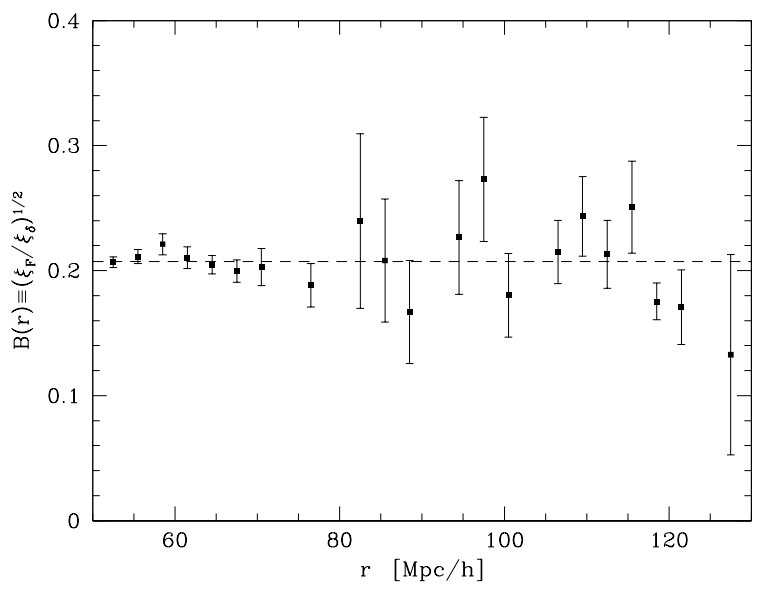

FIG. 5.- The flux bias, $B^{2}(r) \equiv \xi_{F} / \xi_{\delta}$, as a function of scale. While the results are noisy, the bias is consistent with being scale-independent as noted earlier by Slosar et al. (2009).

Slosar et al. (2009) found $B(r)$ was approximately scaleindependent, suggesting a good template for the flux correlation function is the linear theory, real-space, mass correlation function multiplied by an effective bias - shown as the solid line in Figure 4 . Our simulations are consistent with this finding (Figure 5), but since we have less volume our constraints are not as strong.

\subsection{Covariance}

To assign error bars to the points we can use either the boxto-box scatter or a bootstrap scheme (Efron 1982). We tried two different methods for determining the bootstrap errors. The first was simply to draw boxes randomly (with replacement) and compute the average correlation function from the boxes. In the second we randomly assigned each skewer to one of 10 bunches within each simulation and used bootstrap resampling on the full collection of bunches in all of the boxes. Within any individual box the bootstrap errors will drastically underestimate the covariance on large scales, however in the limit that we have a large number of independent

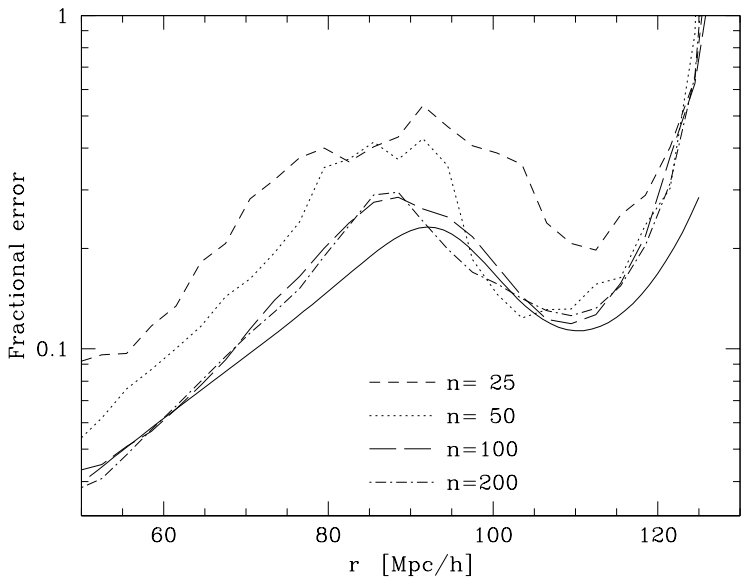

FIG. 6.- The fractional error on $\xi_{F}$ as a function of scale for different choices of sight-line areal density (per sq.deg.). Since the errors can be noisy, we smoothed them over a bin of width $10 h^{-1} \mathrm{Mpc}$. Note the fractional error is larger when $\xi_{F}$ is small, near $r \simeq 90 h^{-1} \mathrm{Mpc}$, and smaller near the peak in $\xi_{F}$ at $r \simeq 110 h^{-1} \mathrm{Mpc}$. The solid line shows the expectation for Gaussian fluctuations.

boxes and the skewer bunches are uncorrelated it will provide a good estimate of the error from the finite survey volume (which we expect to dominate). We find that the box-to-box variance and first bootstrap scheme provided consistent and more stable indicators of the large-scale errors than the second bootstrap. This suggests that with observational data one wants to bootstrap on disjoint regions of the sky rather than on individual quasar sight-lines. It may also be helpful to apply a high-pass filter to make the individual regions more independent (see e.g. Padmanabhan et al. 2009, for discussion), though we do not need to do this in our simulations.

Our 9 boxes cover approximately 1,000 sq.deg., or roughly $10 \%$ of the area planned for BOSS. In this regime the errors scale as area ${ }^{-1 / 2}$, so if all BOSS quasars probed the Lyman$\alpha$ forest at a fixed redshift, and if noise were not an issue, it should achieve errors $\simeq 3 \times$ smaller than those plotted in Figure 6 at similar number densities.

Using this scheme we can look at the dependence of the covariance on the number of lines-of-sight per square degree and on the noise in the individual spectra. Here we vary each of these degrees of freedom independently, allowing us to disentangle the contributions from finite volume, finite sampling and noise. Observationally the only way to achieve a high number density of sources is to go further down the luminosity function, thus at fixed exposure time the signal-to-noise will generally be lower at higher number density. Furthermore the signal-to-noise will usually vary across the spectrum. A detailed study of these issues, or an optimization for a given system, is beyond the scope of this paper.

Figure 6 shows the dependence of the error on $\xi_{F}$ on the density of skewers. Since the error on the error is quite large from our small number of simulations we have smoothed the error with a sliding window of 3 bins in making this figure. Figure 6 shows that increasing the areal density of sight-lines from 25 to 50 per sq.deg. results in better determination of $\xi_{F}$ in the acoustic peak region. However the gains saturate between 50 and 100 quasars per sq.deg. and going to 200 quasars per sq.deg. results in no improvement as the error is dominated by the finite volume surveyed. 
These trends can be readily understood by considering the case of a Gaussian random field which is sampled along a large but finite number of 'skewers'. The observed power spectrum is related to the underlying 3D power spectrum through a window function. For a single k-mode the variance is unaffected by this step, but when considering angularly averaged quantities [e.g. $P(|k|)$ or $\xi(r)$ ] the error is increased (Meiksin \& White 1999; Hamilton et al. 2006). In the limit that the skewers are placed randomly one can show that (see Appendix)

$$
\operatorname{Var}\left[P_{F}\right]=\sum_{j=0}^{3} \frac{v_{j}}{\bar{n}^{j}}
$$

where $\bar{n}$ is the number density of sightlines and the $v_{j}$ involve the power spectrum and its integral in a k shell, $k-\Delta k / 2<$ $|\mathbf{k}|<k+\Delta k / 2$. The lowest order term, $v_{0}=2 P_{F}^{2} / N_{k}$, is the familiar result with $N_{k}$ the number of modes in the shell (Feldman et al. 1994). The ratio $v_{1} / v_{0}$ controls the number density at which we achieve essentially sample variance limited constraints, with the relevant figure of merit depending on the ratio of the achieved line-of-sight (i.e. 2D) quasar number density to a "critical" value (near $0.01 h^{2} \mathrm{Mpc}^{-2}$ ) as described in the Appendix. For observations at $z \sim 2$ this critical number density is between 50-100 quasars per square degree (depending on the redshift range considered), which is where we see diminishing returns in Figure 6.

While quasar counts at such faint limits are quite uncertain, the critical number density corresponds to quasars brighter than a $B$-band magnitude of about 22 . Since it is unlikely that all such quasars in the range $2<z<3$ could be successfully targeted, and since not all of the forest region will be useful for cosmology in all quasars, the relevant sample limits should be fainter. At these magnitudes going one magnitude fainter approximately doubles the number of quasars per square degree (in a fixed, depth $\sim 500 h^{-1} \mathrm{Mpc}$, redshift slice) and going two magnitudes fainter increases the density by a factor $\sim 3.5$.

In the limit of infinite sampling density the error approaches the sample variance limit, which for $\xi(r)$ can be written as

$$
\operatorname{Cov}\left[\xi_{i}, \xi_{j}\right]=\frac{2}{V} \int \frac{d^{3} k}{(2 \pi)^{3}} P_{F}^{2}(k) W_{i}(k) W_{j}(k)
$$

where $W_{i}$ indicates the window function for bin $i$ (Eq.4) and we have assumed the monopole dominates the higher multipoles. The relevant volume is $V=3.8\left(h^{-1} \mathrm{Gpc}\right)^{3}$ and the plotted error in Figure 6 is the square root of the diagonal entries of this matrix. For a large number of skewers the simulationbased errors are in reasonable agreement with the Gaussian prediction in the region of the acoustic peak, however the results are still somewhat noisy. The results become increasingly noisy for the off-diagonal elements of the covariance matrix, so we have not attempted a quantitative comparison. The Gaussian prediction for our $3 h^{-1} \mathrm{Mpc}$ bins is that $\xi$ at the peak ( $r \simeq 110 h^{-1} \mathrm{Mpc}$ ) is correlated with $r=50,90$ and $100 h^{-1} \mathrm{Mpc}$ at the 30, 80 and 90 per cent level.

Figure 7 shows the effects of adding noise to the spectra. We use 100 quasars per sq.deg., since at that areal density the volume is well sampled, and add uncorrelated Gaussian noise to each pixel such that the $S / N$ is unity per $h^{-1} \mathrm{Mpc}$, or very close to unity per $\AA$. It is clear from Figure 7 that noise at this level is a non-trivial contributor to the total error budget at this number density and volume. Recall, as discussed above, that in the absence of binning or finite pixels the white-noise error

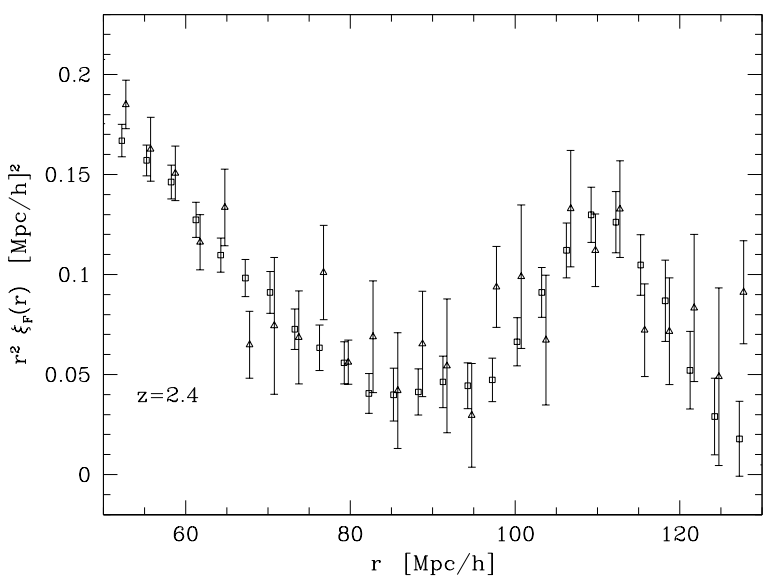

FIG. 7.- The redshift space, flux correlation function for the spectra with no noise (squares) and including noise with $S / N=1$ per $h^{-1} \mathrm{Mpc}$ (which is close to 1 per $\AA$; triangles). The errors are estimated by bootstrapping the 9 boxes.

on $\xi$ at any $r$ is infinite. Since our $r$-bins are larger than our pixels it is this size which tames this infinity, so the precise results depend on the chosen binning.

\subsection{Broad band power \& compensated statistics}

One of the advantages of measuring the correlation function, rather than the power spectrum, is that it is straightforward to compute for even complicated geometries (Slosar et al. 2009). However when estimating the correlation function one needs to pay attention to the mean flux in the survey (equivalent to the mean galaxy density, or "integral constraint" in the case of galaxies). This is not an issue for estimates of the power spectrum, which neatly immunizes high $k$ power from slowly varying signals such as a mean-density or mean-flux error. A (small) misestimate of $\bar{F}$ in the power spectrum leads to a (small) misestimate of the amplitude of $P_{F}$. In the correlation function however an error in the mean flux generates both an additive and multiplicative error,

$$
\xi_{F} \rightarrow \frac{\bar{F}_{\text {true }}^{2}}{\bar{F}_{\text {est }}^{2}} \xi_{F}+\left(1-\frac{\bar{F}_{\text {true }}}{\bar{F}_{\text {est }}}\right)^{2}
$$

and the additive term can swamp the desired correlation function at large scales. If the mean flux evolves with redshift (or distance) the situation is slightly more complicated. However the limit where the mean flux for any pair of points is the same (e.g. the separation of the two points is transverse to the line of sight or the separation vector is much smaller than the characteristic scale over which $F$ varies) is a simple generalization of the above. If $\xi_{F}$ is $\chi$ - or $z$-independent (as it is in our boxes) and the measured mean flux is $\bar{F}$, we have

$$
\xi_{\text {obs }}(r)=\frac{\left\langle F^{2}\right\rangle}{\bar{F}^{2}} \xi_{F}+\left(1+\frac{\left\langle F^{2}\right\rangle}{\bar{F}^{2}}-2 \frac{\langle F\rangle}{\bar{F}}\right)
$$

where $\langle\cdots\rangle$ indicates an average over distance or redshift. The general case is more complex, involving additional correlators of the mean flux and $\delta_{F}$.

To handle this one can either fit out a smoothly varying piece of $\xi_{F}$ (as advocated in e.g. Seo \& Eisenstein 2005, 2007; White 2005, to handle galaxies) or compute a statistic which automatically reduces the sensitivity to slowly varying modes 


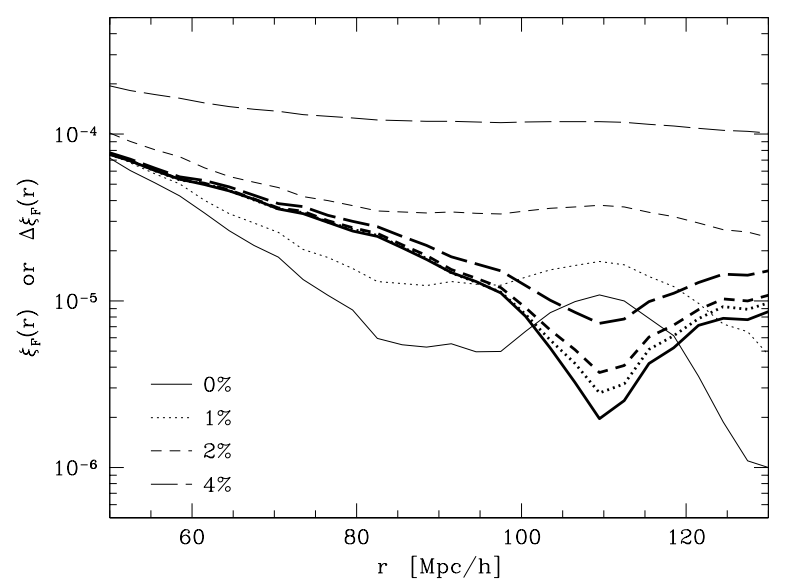

FIG. 8.- The flux correlation function, $\xi_{F}$ (thin lines), and compensated correlation function, $\Delta \xi_{F}$ (thick lines), for situations where the mean flux varies linearly across the box with an end-to-end amplitude of $0,0.01,0.02$ and 0.04 about a fixed mean flux at the box mid-plane.

(e.g. Huff et al. 2007; Padmanabhan et al. 2007b; Xu et al. 2010).

Figure 8 shows the "extra" correlation that is introduced by a mean flux which varies linearly (by $0,1,2$ and 4\%) with distance across the box and that much of this is taken out by

$$
\Delta \xi_{F}(r) \equiv\left\langle\xi_{F}(<r)\right\rangle-\xi_{F}(r)
$$

where the first term indicates the average of the correlation function within $r$ (Huff et al. 2007). Note that in $\Delta \xi_{F}$ the acoustic "peak" becomes an acoustic "dip". We found that the change in $\Delta \xi_{F}$ with increasing mean flux variation is not well fit by a constant multiplicative or constant additive factor. Interpreted as a multiplicative change, the ratio is 1 below $\sim 50 h^{-1} \mathrm{Mpc}$, grows smoothly to a factor of 1.4(4) at the peak, and then drops again beyond the peak for mean flux evolution of 1(4)\% across the box. The larger the mean flux evolution, the broader the effect in $r$ and for all cases the ratio peaks at the acoustic $\operatorname{dip}\left(r \simeq 110 h^{-1} \mathrm{Mpc}\right.$ ) and thus alters the dip location very little. If unaccounted for such evolution would lead to poor fits between the theoretical template and the observations, but the acoustic signal survives in the measurement.

Assuming Eq. (2), to limit the flux evolution to less than $1 \%$ we would need to analyze the spectra in shells of redshift $\Delta z \leq 0.05$ and for $4 \%$ the shell would have to be $\Delta z \leq 0.2$. Alternatively we could imagine including a model for the mean flux evolution in our theoretical prediction or, since we know the mean flux evolution is in the line-of-sight direction it may be possible to isolate this piece by appealing to a model for the angular dependence of $\xi_{F}(\vec{r})$. Since such a model should also handle redshift space distortions we defer this to a future publication.

\section{NON-GRAVITATIONAL CONTRIBUTIONS}

One possible cause for concern, in addition to systematic errors in the measurements themselves, is non-gravitational contributions to the flux correlations. These could arise from hydrodynamic forces, radiative transfer effects, reionization heating or other departures from the simple FGPA assumed thus far (Meiksin 2007). Many of these effects are expected to contribute mostly on small scales, with no power preferentially on the acoustic scale, and should not bias a
BAO measurement (though it may reduce the signal-to-noise of the measurement). Here we investigate two such nongravitational contributions: spatial fluctuations in the ionizing background radiation and spatial fluctuations in the temperature-density relation of the gas as could arise e.g. from (inhomogeneous) HeII reionization. As mentioned previously, fluctuations in the quasar continuum should be less of a concern when we average the cross-spectra from many different quasar sightlines than when we look at the auto-spectra of individual sightlines (Viel et al. 2002; White 2003).

\subsection{Fluctuations in the ionizing radiation}

One possible contributor of large-scale power is fluctuations in the UV background field or the photo-ionization rate $(\Gamma)$. Since the attenuation length of the IGM at $z \sim 2-3$ is $\mathcal{O}(100 \mathrm{Mpc})$, and the background is thought to be dominated by rare sources (QSOs), $\Gamma$ may have spatial structure on large scales (Zuo 1992; Fardal \& Shull 1993; Gnedin \& Hamilton 2002; Meiksin \& White 2004; Croft 2004; McDonald et al. 2005). Assuming the IGM is in photo-ionization equilibrium the optical depth, $\tau \propto \Gamma^{-1}$. Slosar et al. (2009) investigated the effects of a fluctuating photo-ionization rate in a highly simplified model. We follow Slosar et al. (2009) in assuming that the ionizing background field is dominated by light from quasars, but extend the analysis to consider diagnostics of these additional fluctuation sources.

We place our quasars at random within the volume with luminosities drawn from a luminosity function

$$
\Phi \propto \frac{1}{\left(L / L_{\star}\right)^{-\alpha}+\left(L / L_{\star}\right)^{-\beta}}
$$

with $\alpha=-3.31$ and $\beta=-1.09$ (Croom et al.2004). Each QSO is assumed to emit isotropically with constant luminosity $L$, so the contribution to the photo-ionization rate from the $i$ th QSO at distance $r_{i}$ can be taken to be

$$
\Gamma_{i} \propto L_{i} \frac{e^{-r_{i} / r_{0}}}{4 \pi r_{i}^{2}},
$$

neglecting finite lifetimes or light-cone effects (see Croft 2004, for further discussion of these issues). Here $r_{0} \simeq$ $\mathcal{O}\left(100 h^{-1} \mathrm{Mpc}\right)$ is the 'attenuation length' of the IGM, which is a rapidly decreasing function of redshift.

Meiksin \& White (2004) find, for a $\Lambda$ CDM cosmology, that the (comoving) attenuation length is approximately

$$
r_{0} \simeq 2 \times 10^{4}(1+z)^{-3.2} h^{-1} \mathrm{Mpc}
$$

for $2.75<z<5.5$, with diffuse gas and Lyman Limit Systems contributing approximately equally. This gives a comoving attenuation length of $r_{0} \approx 200 h^{-1} \mathrm{Mpc}$ at $z=3$, with larger values at lower $z$. This result appears to agree with more recent measurements, for example the recent measurement of Prochaska et al. (2009) at higher $z$ agree with the results above where they overlap. In Slosar et al. (2009) a value of $r_{0}=100 \mathrm{~h}^{-1} \mathrm{Mpc}$ was chosen, to emphasize effects at the acoustic scale. Here we use $r_{0} \approx 200 h^{-1} \mathrm{Mpc}$, which is closer to the value inferred above, neglecting the dependence on redshift. Increasing $r_{0}$ suppresses the photo-ionization fluctuation auto-correlation function on small scales while increasing it on the (large) scales of interest (in agreement with analytic arguments; Zuo 1992). This makes the impact of a fixed-rms $\Gamma$ fluctuation larger at the acoustic scale, further decreasing 
the contrast of the acoustic peak. For simplicity we have made the approximation that $r_{0}$ is the same for all quasars.

To the particular realization of the QSO component we add a uniform piece (to model the emission from faint AGN, galaxies and the IGM itself; Meiksin 2007) such that the rms fluctuation of the total is $10 \%$ and divide our original $\tau$ in every pixel by the ionization rate at that location. The overall normalization is rescaled to match the mean flux, $\bar{F}$.

Ideally we would have used the positions of the dark matter halos for the sources of our photo-ionizing flux. However we do not have halo information for the Roadrunner simulations. To test for the possible effect of quasar clustering on our $\Gamma(\vec{x})$ we looked at another set of simulations, run using a high force-resolution TreePM code (White 2002) in $1.25 h^{-1} \mathrm{Gpc}$ cubes, for which we did have stored halo catalogs. Using the model of Croton (2009) we assigned quasar luminosities to each halo, with the lowest luminosity quasar having $L / L_{\star} \simeq 2 \%$. The resulting luminosity function and clustering agree well with observations at $z \sim 2-3$. For both these 'quasars', and a set with randomized positions but the same luminosities, we computed $\Gamma(\vec{x})$ (as above) for $10^{4}$ randomly placed skewers and hence $\xi_{\Gamma}$. Comparison of $\xi_{\Gamma}$ for the haloproduced $\Gamma(\vec{x})$ and the position-randomized $\Gamma(\vec{x})$ gives us a measure of the effect of source clustering. There is significant variation, box-to-box, in $\xi_{\Gamma}$ but the mean at $r \simeq 100 h^{-1} \mathrm{Mpc}$ is increased by quasar clustering by $\sim 30(20) \%$ when $r_{0}=$ $100(200) h^{-1} \mathrm{Mpc}$. These are small increases in $\xi_{\Gamma}$, but the fact that the halos trace the density field induces correlations between $\Gamma$ and $\delta_{m}$ which are missed by our random-position approximation and which we cannot adequately probe. It remains an open question whether matter-correlated source clustering is important for $\Gamma$ fluctuations. We also note that $r_{0}$ is becoming an appreciable fraction of the simulation box length in our main simulations, raising questions of numerical convergence. It would be nice to confirm and extend this analysis with larger simulations including the halo catalogs, and we plan to return to this in a future publication.

\subsection{Fluctuations in the IGM temperature}

In addition to the ionizing background fluctuations described above, the Universe undergoes HeII reionization by bright QSOs around $z \sim 3$ (Meiksin 2007). This event can heat regions of the IGM by as much as $25,000 \mathrm{~K}$, with large temperature fluctuations on 50 Mpc scales (McQuinn et al. 2009). The large QSO bubbles responsible for HeII reionization are essentially uncorrelated with the overdensity at a given location and no value of $\delta$ is preferentially ionized at a given time during HeII reionization (McOuinn et al. 2009). This leads to significant scatter in $T$ at fixed $\delta$ which is approximately "random".

In order to mock up such a situation in as simple a manner as possible, we randomly throw 1,000 centers within the box and assign each a radius of influence equal to the mean intercenter separation and a temperature drawn from a log-normal of mean $2 \times 10^{4} \mathrm{~K}$ and $\sigma_{\ln T}=1$. The temperature at mean density at any point in the box is then the weighted sum of these values, with a Gaussian weight depending on distance from each center. This gives fluctuations in the temperature at mean density which are large, but highly coherent over large distances and smoothly varying.

\subsection{Effects on $\Delta \xi_{F}$}

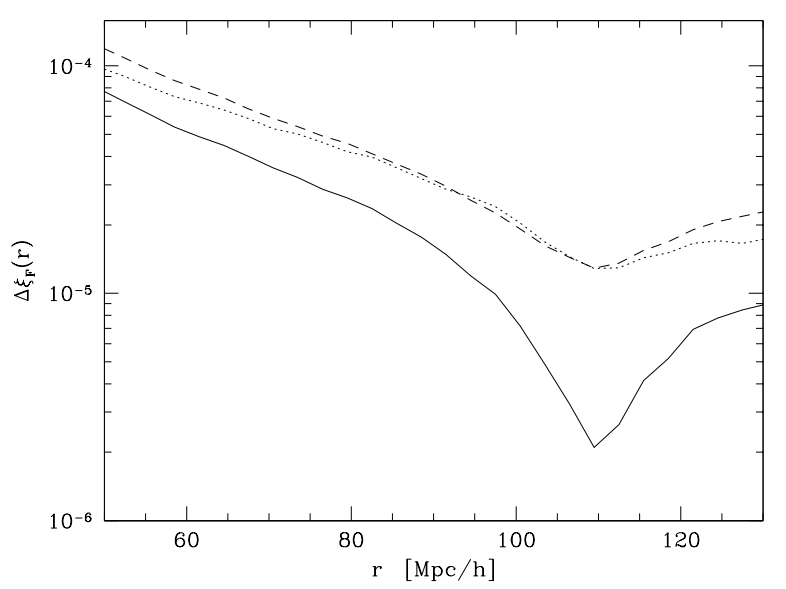

FIG. 9.- The compensated flux correlation function, $\Delta \xi_{F}$, vs. scale for our fiducial model (solid), a model with $\Gamma$-fluctuations as described in the text (dotted) and a model with fluctuating $T_{0}$ (dashed). The large "extra" power which dominates $\xi_{F}$ in the latter two models is removed in $\Delta \xi_{F}$, and the acoustic signal remains visible though reduced in contrast.

Both the fluctuations in the photo-ionization rate and modulation of the mean temperature give rise to large changes in the correlation function, similar to what was seen for an evolving $\bar{F}(\$ 3.4)$. As there, much (but not all) of the 'extra' signal is smooth and can be removed by considering a compensated statistic. Figure 9 shows the effect of $\Gamma$ and $T_{0}$ fluctuations on $\Delta \xi_{F}$.

\subsection{Diagnostics}

Diagnostics of these non-gravitational contributions can be found in the higher moments of the flux (Zaldarriaga et al. 2001b; Fang \& White 2004) and all indications are that the forest is dominated by gravitational instability on large scales. However, the errors on such measurements are still large enough that the issue is not settled.

The existing measurements of inter-scale correlations were done on individual spectra by computing the cross-power between a power of the (band-pass) filtered flux field and the original flux field. The auto- and cross-power spectra can be related to the power spectrum, bispectrum and trispectrum of the flux. Gravitational instability induces a positive correlation between large- and small-scale density fluctuations, which leads to a negative correlation coefficient, $C(k)$, between the filtered field and the original flux.

In the absence of non-gravitational contributions $C(k) \approx 0$ on scales smaller than the cut-off in the band-pass filter and quickly tends to negative values on larger scales. The value of $C(k \rightarrow 0)$ depends primarily on the width of the band-pass filter, being more negative the wider the filter. Existing observations of a handful of high $\mathrm{S} / \mathrm{N}$, high resolution spectra are consistent with the structure produced in gravity-only simulations using the FGPA.

We find that in the presence of $\Gamma$-fluctuations the crosscorrelation, $C(k)$, is altered by a small amount at $k^{-1} \sim$ $100 h^{-1} \mathrm{Mpc}$ for band-passes focused on small scales. Such an alteration would have been totally unmeasurable in the existing analyses, and whether it is observable in the presence of unknown continuum structure and other observational nonidealities remains in doubt. The effects of large-scale coherent fluctuations in $T_{0}$ are also quite small in this statistic and would not have been seen in existing analyses. 


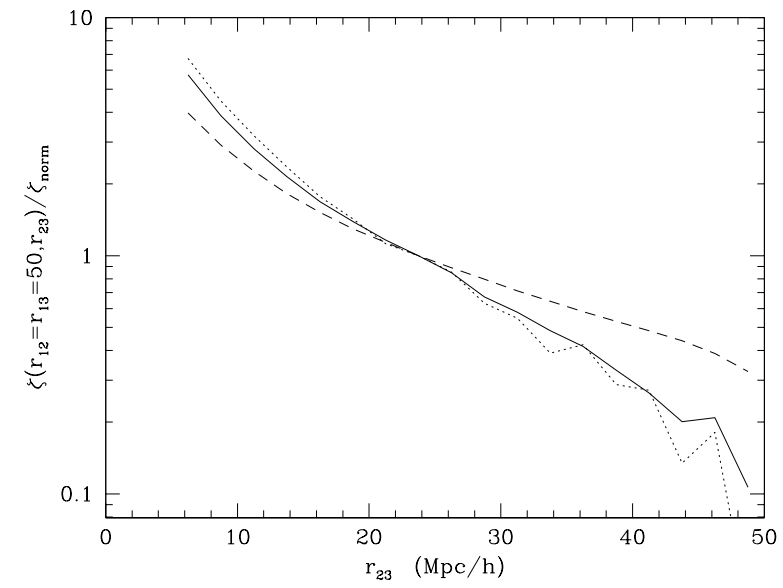

FIG. 10.- The 3-point flux correlation function in configuration space for isosceles triangles with $r_{12}=r_{13}=50 h^{-1} \mathrm{Mpc}$ vs. $r_{23}$ for the fiducial model (solid), a model with $\Gamma$-fluctuations as described in the text (dotted) and a model with fluctuating $T_{0}$ (dashed). An overall amplitude has been removed by normalizing to $\zeta$ with $r_{23}=r_{12} / 2$. Note that the model with fluctuating $T_{0}$ shows a different scale dependence than the other models.

The basic idea of measuring the higher order correlations and looking for gravitationally induced signatures remains valid however. With upcoming BOSS data as a motivation, we here present preliminary results on the large-scale 3-point cross-correlation function along three different lines-of-sight. Such a statistic will be less susceptible to noise and continuum fluctuations than the measurement discussed above.

There are many possible configurations for which we could evaluate the flux 3-point function, and we could evaluate it in configuration or Fourier space. We choose here to use an isosceles triangle in configuration space. Particularly for $T_{0}$ fluctuations from He reionization, we might expect configurations where two vertices are in one bubble and one is in another to show a large non-gravitational signal. Figure 10 shows the 3-point flux correlation function in configuration space for isosceles triangles with $r_{12}=r_{13}=50 h^{-1} \mathrm{Mpc}$ vs. $r_{23}$. Our simulations show that there is a large variance in the 3-point function from box to box, and smaller equationsof-state, $\gamma$, lead to a steeper dependence of $\zeta$ on $r_{23}$. The model with fluctuating $\Gamma$ cannot be distinguished from pure gravity models with $1<\gamma<1.5$ based on the shape dependence shown here. Similarly increasing the temperature uniformly to $3 \times 10^{4} \mathrm{~K}$ gives a result close to the solid line in Figure 10. However, the model with $T_{0}$ fluctuations shows a much flatter dependence on $r_{23}$ than the others, enabling a test of the non-gravitational nature of the fluctuations. We have chosen to plot $r_{12}=50 h^{-1} \mathrm{Mpc}$ since on smaller scales the 3-point function is very similar for all of the models (as expected) while on large scales the results become very noisy though the model differences appear to be accentuated. In principle, a survey like BOSS would be able to detect the difference with high significance. Whether these differences can be determined in the presence of other sources of noise and modeling uncertainty, how the signals scale with the model parameters and what the best higher-order statistic is, all need to be investigated and we hope to return to this in future work.

\section{DISCUSSION AND CONCLUSIONS}

Baryon acoustic oscillations have become one of the premier methods for determining cosmological distances and hence the expansion history of the Universe. The structure in the spectrum of distant quasars, which is thought to trace the structure of the IGM at near mean density, has been suggested as a relatively cheap method for measuring BAO at high redshift. Motivated by the upcoming BOSS experiment, and the availability of the world's fastest supercomputer, "Roadrunner", we have performed a set of ultra-large particle-mesh simulations in order to study the BAO signature in the intergalactic medium.

From the density and velocity fields we have used the "fluctuating Gunn-Peterson approximation" (FGPA) to produce mock Lyman- $\alpha$ spectra. These simulations are the first to be able to cover the large volume necessary to constrain the acoustic scale while simultaneously resolving the Jeans scale of the gas, and the small-scale power and pixel distribution in our mock spectra approximately match those seen in observations at $z \simeq 2.5$. We anticipate that these mock spectra will be very useful in testing pipelines, calibrating analysis tools and planning future projects so we have made them publicly available屯.

In the simulations the Lyman- $\alpha$ flux follows the mass fluctuations on large scales, with negligible scale-dependent bias (Slosar et al. 2009). This is in some sense to be expected. We have assumed the FGPA in producing our mock spectra and within this approximation the flux is a highly non-trivial but deterministic function of the underlying density field.

Since the small-scale statistics in our simulations approximately match observations, we are able to make a reasonable estimate of the error in the flux auto-correlation function. We discuss the scaling of the error with the number of quasar sightlines and the noise in each spectrum. In the limit of low noise and many sightlines the error on the correlation function is well approximated by the Gaussian expression.

We have presented preliminary investigations of an evolving mean flux, fluctuations in the photo-ionization rate and HeII reionization which generate "extra" power on the acoustic scale and reduce the contrast of the acoustic peak. Gravitational instability produces a well-defined pattern of higher order correlations which is not obeyed by non-gravitational contributions such as the above, allowing (in principle) a diagnostic of non-gravitational physics in the forest. As an example, we demonstrate that the 3-point cross-correlation function in models with HeII reionization has a different scale dependence than the 3-point function in gravity-only simulations, regardless of the equation-of-state assumed in the latter.

MW thanks Shirley Ho, Matt McQuinn, Avery Meiksin and Anze Slosar for conversations on the Lyman- $\alpha$ forest. The simulations used in this work were run at LANL under the Roadrunner Open Science Program. Part of the analysis was done under the Los Alamos Institutional Computing Program. We thank the LANL Roadrunner support team for their outstanding effort in helping complete the simulation runs for this project. The analysis also made use of the computing resources of the National Energy Research Scientific Computing Center and the Laboratory Research Computing project at Lawrence Berkeley National Laboratory. MW is supported by the NASA and the DoE. Part of this research was supported by the DOE under contract W-7405-ENG-36. DD, PF, SH,

\footnotetext{
${ }^{4}$ http://mwhite.berkeley.edu
} 
KH, ZL, AP acknowledge support from the LDRD program at Los Alamos National Laboratory.

\section{APPENDIX}

\section{SCALING WITH NUMBER DENSITY}

The distribution of quasars on the sky determines at what places we sample the underlying density field. As the number density of quasars increases so does the number of samples with which we can constrain $P(k)$ or $\xi(r)$, and hence the fidelity of the measurement, but eventually we are limited by the available modes within the survey volume. Here we describe how that limit is approached for a Gaussian field, which is a reasonable approximation on large scales (see $\$ 3.3$ ). has

To begin we consider a large periodic volume of side length $L$ and volume (or area in $2 \mathrm{D}$ ) $V$. Our Fourier transform convention

$$
\delta(\mathbf{k})=V^{-1} \int_{V} d V \delta(\mathbf{r}) \exp [i \mathbf{k} \cdot \mathbf{r}] \quad, \quad \delta(\mathbf{x})=\sum_{\mathbf{k}} \delta(\mathbf{k}) \exp [-i \mathbf{k} \cdot \mathbf{r}]
$$

both dimensionless and $P(k)=V\left\langle|\delta(\mathbf{k})|^{2}\right\rangle$. To model the finite sampling we multiply the density field, $\delta(\mathbf{x})$, by a window function, $w(\mathbf{x})$, which is non-zero only where the field is observed, leading to

$$
P(\mathbf{k})=\sum_{\mathbf{k}^{\prime}} P\left(\mathbf{k}^{\prime}\right)\left|w_{\mathbf{k}-\mathbf{k}^{\prime}}\right|^{2}
$$

We shall approximate $w(\mathbf{x})$ as a sum of (randomly distributed) 2D $\delta$-functions, or $w(\mathbf{k})$ as a sum of plane waves. If we average in a shell $S_{i}$ with $k_{i}-\Delta k / 2<|\mathbf{k}|<k_{i}+\Delta k / 2$ we obtain

$$
\widehat{P}_{i} \equiv \frac{1}{N_{i}} \sum_{\mathbf{k} \in S_{i}} \sum_{\mathbf{k}^{\prime}, \mathbf{k}^{\prime \prime}} \delta_{\mathbf{k}^{\prime}}^{\star} \delta_{\mathbf{k}^{\prime \prime}} w_{\mathbf{k}-\mathbf{k}^{\prime}}^{\star} w_{\mathbf{k}-\mathbf{k}^{\prime \prime}}
$$

with mean

$$
\left\langle\widehat{P}_{i}\right\rangle=\frac{1}{N_{i}} \sum_{\mathbf{k} \in S_{i}} \sum_{\mathbf{k}^{\prime}} P\left(k^{\prime}\right)\left|w_{\mathbf{k}-\mathbf{k}^{\prime}}\right|^{2}
$$

and covariance

$$
\operatorname{Cov}\left[\widehat{P}_{i}, \widehat{P}_{j}\right]=\frac{2}{N_{i} N_{j}} \sum_{\mathbf{k}_{1} \in S_{i}} \sum_{\mathbf{k}_{2} \in S_{j}} \sum_{\mathbf{k}^{\prime}, \mathbf{k}^{\prime \prime}} P\left(k^{\prime}\right) P\left(k^{\prime \prime}\right) w_{\mathbf{k}_{2}-\mathbf{k}^{\prime}} w_{\mathbf{k}^{\prime}-\mathbf{k}_{1}} w_{\mathbf{k}_{1}-\mathbf{k}^{\prime \prime}} w_{\mathbf{k}^{\prime \prime}-\mathbf{k}_{2}}
$$

where $N_{i}$ is the number of modes in the shell and we have explicitly assumed $P$ depends only on $|\mathbf{k}|$ (e.g. we have neglected redshift space distortions).

If $w(\mathbf{x})$ is a sum of $n_{\text {los }} \delta$-functions, whose positions we average over, the product of $w(\mathbf{k})$ 's in Eq. A5 becomes a sum of Kronecker $\delta$ 's. There are 4 kinds of terms (depending on the number of points which coincide) which scale as $1,1 / n_{\text {los }}, 1 / n_{\text {los }}^{2}$ and $1 / n_{\text {los }}^{3}$ respectively. In $2 \mathrm{D}$ in the continuum limit and for narrow bins the result becomes

$$
\operatorname{Var}\left[\widehat{P}_{i}\right]=\frac{2}{N_{i}} P\left(k_{i}\right)^{2}\left(1+\frac{\sigma^{2}}{\bar{n} P\left(k_{i}\right)}\right)^{2}
$$

where $\sigma^{2}$ is the density variance and $\bar{n}$ is the line-of-sight number density. The ratio of the terms going as 1 and $1 / \bar{n}$ determines at what number density we approach the sample variance limit.

In 3D the situation is slightly more complex because the window function has no structure in the line-of-sight direction, which gives a $\delta$-function in $k_{\text {los }}$, and the spherical average involved in $\widehat{P}_{i}$ gives more structure to the expression. In the continuum limit, the approach to the sample variance limit is governed by the ratic $\sqrt{5}$

$$
\frac{v_{1}}{\bar{n} v_{0}} \rightarrow \frac{\pi}{\bar{n} k_{i} P\left(k_{i}\right)} \int \frac{d^{3} \mathbf{k}}{(2 \pi)^{3}} P(k) W(k)
$$

where the $v_{j}$ are defined in Eq. (6) and $W(k)$ is unity for $|\mathbf{k}|<k_{i}$ and $k_{i} /|\mathbf{k}|$ for $|\mathbf{k}|>k_{i}$. For $\Lambda$ CDM cosmologies the integral is $\mathcal{O}(0.01)$ on the scales of interest. The additional variance depends then upon $\bar{n}_{\text {eff }} P\left(k_{i}\right) / P_{0}$ where $\bar{n}_{\text {eff }}=k \bar{n}_{2 D} \sim \bar{n} / \lambda$ and we have included the $P_{0}$ factor to indicate that the ratio is independent of the normalization of $P(k)$. We note that this expression is qualitatively similar to, but not the same as, the expression given in McDonald \& Eisenstein (2007, Eq. 13).

For small $k_{i}$ the integral above can be approximated by the 1D power spectrum (an approximation that becomes worse as $k \rightarrow \infty)$. With that approximation the ratio can be written as $\bar{n}_{\text {crit }} / \bar{n}$, with the critical (2D) number density being

$$
\bar{n}_{\text {crit }} \equiv \frac{\Delta_{1 D}^{2}\left(k_{i}\right)}{k_{i} P\left(k_{i}\right) / \pi} \quad \text { where } \quad \Delta_{1 D}^{2}\left(k_{i}\right) \equiv k_{i} \int_{k_{i}}^{\infty} \frac{d^{3} \mathbf{k}}{(2 \pi)^{3}} \frac{P(k)}{k}
$$

${ }^{5}$ Including the next-order correction involving $v_{2}$ is important for quanti-

tative accuracy. 
which depends on the shape of the power spectrum but not its normalization. As expected, cosmologies with more smallscale power require a higher number density of skewers to reach the sample variance dominated limit. For our model $\bar{n}_{\text {crit }} \sim$ $0.01 h^{2} \mathrm{Mpc}^{-2}$, in agreement with the trends seen in Figure 6

\section{REFERENCES}

Bharadwaj, S. 1996, ApJ, 472, 1

Blake, C., Collister, A., Bridle, S., \& Lahav, O. 2007, MNRAS, 374, 1527

Chang, T.-C., Pen, U.-L., Peterson, J. B., \& McDonald, P. 2008, Physical Review Letters, 100, 091303

Cole, S., Percival, W. J., Peacock, J. A., Norberg, P., Baugh, C. M., Frenk, C. S., Baldry, I., Bland-Hawthorn, J., Bridges, T., Cannon, R., Colless, M., Collins, C., Couch, W., Cross, N. J. G., Dalton, G., Eke, V. R., De Propris, R., Driver, S. P., Efstathiou, G., Ellis, R. S., Glazebrook, K., Jackson, C., Jenkins, A., Lahav, O., Lewis, I., Lumsden, S., Maddox, S., Madgwick, D., Peterson, B. A., Sutherland, W., \& Taylor, K. 2005, MNRAS, 362, 505

Crocce, M., \& Scoccimarro, R. 2008, Phys. Rev. D, 77, 023533

Croft, R. A. C. 2004, ApJ, 610, 642

Croft, R. A. C., Weinberg, D. H., Bolte, M., Burles, S., Hernquist, L., Katz, N., Kirkman, D., \& Tytler, D. 2002, ApJ, 581, 20

Croft, R. A. C., Weinberg, D. H., Katz, N., \& Hernquist, L. 1998, ApJ, 495, 44

Croom, S. M., Smith, R. J., Boyle, B. J., Shanks, T., Miller, L., Outram, P. J., \& Loaring, N. S. 2004, MNRAS, 349, 1397

Croton, D. J. 2009, MNRAS, 394, 1109

Dehnen, W. 2001, MNRAS, 324, 273

Efron, B. 1982, The Jackknife, the Bootstrap and other resampling plans, ed. B. Efron

Eisenstein, D. J., \& Hu, W. 1998, ApJ, 496, 605

Eisenstein, D. J., Seo, H.-J., \& White, M. 2007, ApJ, 664, 660

Eisenstein, D. J., Zehavi, I., Hogg, D. W., Scoccimarro, R., Blanton, M. R., Nichol, R. C., Scranton, R., Seo, H.-J., Tegmark, M., Zheng, Z., Anderson, S. F., Annis, J., Bahcall, N., Brinkmann, J., Burles, S., Castander, F. J., Connolly, A., Csabai, I., Doi, M., Fukugita, M., Frieman, J. A., Glazebrook, K., Gunn, J. E., Hendry, J. S., Hennessy, G., Ivezić, Z., Kent, S., Knapp, G. R., Lin, H., Loh, Y.-S., Lupton, R. H., Margon, B., McKay, T. A., Meiksin, A., Munn, J. A., Pope, A., Richmond, M. W., Schlegel, D., Schneider, D. P., Shimasaku, K., Stoughton, C., Strauss, M. A., SubbaRao, M., Szalay, A. S., Szapudi, I., Tucker, D. L., Yanny, B., \& York, D. G. 2005, ApJ, 633, 560

Fang, T., \& White, M. 2004, ApJ, 606, L9

Fardal, M. A., \& Shull, J. M. 1993, ApJ, 415, 524

Feldman, H. A., Kaiser, N., \& Peacock, J. A. 1994, ApJ, 426, 23

Gnedin, N. Y., \& Hamilton, A. J. S. 2002, MNRAS, 334, 107

Gnedin, N. Y., \& Hui, L. 1998, MNRAS, 296, 44

Habib, S., Pope, A., Lukić, Z., Daniel, D., Fasel, P., Desai, N., Heitmann, K., Hsu, C., Ankeny, L., Mark, G., Bhattacharya, S., \& Ahrens, J. 2009, Journal of Physics Conference Series, 180, 012019

Hamilton, A. J. S. 1992, ApJ, 385, L5

Hamilton, A. J. S., Rimes, C. D., \& Scoccimarro, R. 2006, MNRAS, 371, 1188

Heitmann, K., Higdon, D., White, M., Habib, S., Williams, B. J., Lawrence, E., \& Wagner, C. 2009, ApJ, 705, 156

Heitmann, K., White, M., Wagner, C., Habib, S., \& Higdon, D. 2008, ArXiv e-prints

Huff, E., Schulz, A. E., White, M., Schlegel, D. J., \& Warren, M. S. 2007, Astroparticle Physics, 26, 351

Hui, L., \& Gnedin, N. Y. 1997, MNRAS, 292, 27

Hütsi, G. 2006, A\&A, 449, 891

Jarosik, N., Bennett, C. L., Dunkley, J., Gold, B., Greason, M. R., Halpern, M., Hill, R. S., Hinshaw, G., Kogut, A., Komatsu, E., Larson, D., Limon, M., Meyer, S. S., Nolta, M. R., Odegard, N., Page, L., Smith, K. M., Spergel, D. N., Tucker, G. S., Weiland, J. L., Wollack, E., \& Wright, E. L. 2010, ArXiv e-prints

Kaiser, N. 1987, MNRAS, 227, 1

Kim, T.-S., Bolton, J. S., Viel, M., Haehnelt, M. G., \& Carswell, R. F. 2007, MNRAS, 382, 1657

Kim, T.-S., Viel, M., Haehnelt, M. G., Carswell, R. F., \& Cristiani, S. 2004, MNRAS, 347, 355

Lidz, A., Faucher-Giguere, C. ., Dall'Aglio, A., McQuinn, M., Fechner, C., Zaldarriaga, M., Hernquist, L., \& Dutta, S. 2009, ArXiv e-prints
Mandelbaum, R., McDonald, P., Seljak, U., \& Cen, R. 2003, MNRAS, 344, 776

Matsubara, T. 2008, Phys. Rev. D, 77, 063530

McDonald, P. 2003, ApJ, 585, 34

McDonald, P., \& Eisenstein, D. J. 2007, Phys. Rev. D, 76, 063009

McDonald, P., Miralda-Escudé, J., Rauch, M., Sargent, W. L. W., Barlow, T. A., \& Cen, R. 2001, ApJ, 562, 52

McDonald, P., Miralda-Escudé, J., Rauch, M., Sargent, W. L. W., Barlow,

T. A., Cen, R., \& Ostriker, J. P. 2000, ApJ, 543, 1

McDonald, P., Seljak, U., Burles, S., Schlegel, D. J., Weinberg, D. H., Cen,

R., Shih, D., Schaye, J., Schneider, D. P., Bahcall, N. A., Briggs, J. W.,

Brinkmann, J., Brunner, R. J., Fukugita, M., Gunn, J. E., Ivezić, Ž., Kent,

S., Lupton, R. H., \& Vanden Berk, D. E. 2006, ApJS, 163, 80

McDonald, P., Seljak, U., Cen, R., Bode, P., \& Ostriker, J. P. 2005, MNRAS, 360,1471

McQuinn, M., Lidz, A., Zaldarriaga, M., Hernquist, L., Hopkins, P. F.,

Dutta, S., \& Faucher-Giguère, C.-A. 2009, ApJ, 694, 842

Meiksin, A., \& White, M. 1999, MNRAS, 308, 1179

-. 2001, MNRAS, 324, 141

-. 2004, MNRAS, 350, 1107

Meiksin, A., White, M., \& Peacock, J. A. 1999, MNRAS, 304, 851

Meiksin, A. A. 2007, ArXiv e-prints

Norman, M. L., Paschos, P., \& Harkness, R. 2009, ArXiv e-prints

Padmanabhan, N., Schlegel, D. J., Seljak, U., Makarov, A., Bahcall, N. A.,

Blanton, M. R., Brinkmann, J., Eisenstein, D. J., Finkbeiner, D. P., Gunn,

J. E., Hogg, D. W., Ivezić, Ž., Knapp, G. R., Loveday, J., Lupton, R. H.,

Nichol, R. C., Schneider, D. P., Strauss, M. A., Tegmark, M., \& York,

D. G. 2007a, MNRAS, 378, 852

Padmanabhan, N., White, M., \& Eisenstein, D. J. 2007b, MNRAS, 376, 1702

Padmanabhan, N., White, M., Norberg, P., \& Porciani, C. 2009, MNRAS, 397, 1862

Peebles, P. J. E., \& Yu, J. T. 1970, ApJ, 162, 815

Percival, W. J., Cole, S., Eisenstein, D. J., Nichol, R. C., Peacock, J. A., Pope, A. C., \& Szalay, A. S. 2007, MNRAS, 381, 1053

Percival, W. J., Reid, B. A., Eisenstein, D. J., Bahcall, N. A., Budavari, T., Frieman, J. A., Fukugita, M., Gunn, J. E., Ivezic, Z., Knapp, G. R., Kron, R. G., Loveday, J., Lupton, R. H., McKay, T. A., Meiksin, A., Nichol, R. C., Pope, A. C., Schlegel, D. J., Schneider, D. P., Spergel, D. N., Stoughton, C., Strauss, M. A., Szalay, A. S., Tegmark, M., Vogeley, M. S., Weinberg, D. H., York, D. G., \& Zehavi, I. 2009, ArXiv e-prints Prochaska, J. X., Worseck, G., \& O'Meara, J. M. 2009, ArXiv e-prints Ricotti, M., Gnedin, N. Y., \& Shull, J. M. 2000, ApJ, 534, 41

Schaye, J., Theuns, T., Rauch, M., Efstathiou, G., \& Sargent, W. L. W. 2000, MNRAS, 318,817

Schlegel, D., White, M., \& Eisenstein, D. 2009, ArXiv e-prints

Seo, H., \& Eisenstein, D. J. 2005, ApJ, 633, 575

-. 2007, ApJ, 665, 14

Slosar, A., Ho, S., White, M., \& Louis, T. 2009, Journal of Cosmology and Astro-Particle Physics, 10, 19

Sunyaev, R. A., \& Zeldovich, Y. B. 1970, Ap\&SS, 7, 3

Theuns, T., Schaye, J., Zaroubi, S., Kim, T.-S., Tzanavaris, P., \& Carswell, B. 2002, ApJ, 567, L103

Viel, M., Matarrese, S., Mo, H. J., Haehnelt, M. G., \& Theuns, T. 2002, MNRAS, 329, 848

White, M. 2002, ApJS, 143, 241

White, M. 2003, in The Davis Meeting On Cosmic Inflation [astro-ph/0305474]

-. 2005, Astroparticle Physics, 24, 334

White, M., \& Cohn, J. D. 2002, American Journal of Physics, 70, 106

Xu, X., White, M., Padmanabhan, N., Eisenstein, D., Eckel, J., Mehta, K., Metchnik, M., Pinto, P., \& Seo, H. 2010, ArXiv e-prints

Zaldarriaga, M., Hui, L., \& Tegmark, M. 2001a, ApJ, 557, 519

Zaldarriaga, M., Seljak, U., \& Hui, L. 2001b, ApJ, 551, 48

Zuo, L. 1992, MNRAS, 258, 45 